\title{
Status of the LHCb Magnet System
}

\author{
J. André, P. Charra, W. Flegel, P. A. Giudici, O. Jamet, P. Lançon, M. Losasso, F. Rohner, and C. Rosset
}

\begin{abstract}
The LHCb experiment focuses on the precision measurement of $\mathrm{CP}$ violation and rare decays in the $B$-meson system. It plans to operate with an average luminosity of $2 \times 10^{32} \mathrm{~cm}^{-2} \mathrm{~s}^{-1}$, which should be obtained from the beginning of the LHC operation. The LHCb detector exploits the forward region of the pp collisions at the LHC collider. It requires a single-arm spectrometer for the separation and momentum measurement of the charged particles with a large dipole magnet of a free aperture of $\pm 300 \mathrm{mrad}$ horizontally and $\pm \mathbf{2 5 0}$ mrad vertically. The magnet is designed for a total integrated field of $4 \mathrm{Tm}$. The pole gap is 2.2 to $3.5 \mathrm{~m}$ vertically (the direction of the field) and 2.6 to $4.2 \mathrm{~m}$ horizontally. The overall length of the magnet (in beam direction) is $5 \mathrm{~m}$ and its total weight about $1500 \mathrm{t}$. The power dissipation in the aluminum coils will be 4.2 MW. The magnet yoke is constructed from low carbon steel plates of $100 \mathrm{~mm}$ thickness. The maximum weight of one plate does not exceed $25 \mathrm{t}$. The coils are wound from large hollow aluminum conductor of $50 \mathrm{~mm} \times 50 \mathrm{~mm}$ cross-section with a central cooling channel of $25 \mathrm{~mm}$ diameter for the pressurized demineralized water. Each of the two coils is composed of 15 monolayer pancakes of 15 turns per pancake. To reach good field quality the coils are bent by $45^{\circ}$ toward the gap along the horizontal aperture of $\pm 300 \mathrm{mrad}$ and the pole pieces have large shims. The underlying magnet design, its present status and milestones will be reviewed.
\end{abstract}

Index Terms-Large sloping gap, resistive dipole magnet for physics experiments.

\section{INTRODUCTION}

$\mathbf{T}$ HE LHCb experiment (b for beauty) at the Large Hadron Collider (LHC) at CERN exploits the forward peaking of beauty production in high-energy proton-proton collisions. This leads to a fixed-target-like open structure of the experimental set-up with a large dipole magnet for the charged particle separation and momentum measurement. The general layout of the LHCb experiment in the underground hall is shown in Fig. 1. The center of the dipole magnet is $5.3 \mathrm{~m}$ downstream of the interaction point.

The detector acceptance requires a free aperture of $\pm 300 \mathrm{mrad}$ horizontally and $\pm 250 \mathrm{mrad}$ vertically (the direction of the field). Tracking detectors in and near the magnetic field have to provide momentum measurements with a precision of about $0.4 \%$ for momenta up to $200 \mathrm{GeV} / \mathrm{c}$. This demands an integrated field of $4 \mathrm{Tm}$ for tracks originating near the primary interaction point. A good field uniformity along the transverse coordinate is needed for the muon trigger. This led to a magnet design with resistive saddle-shaped coils in a window-frame yoke with sloping poles. The design is laid down in the Technical Design Report [1]. This design replaces the originally proposed magnet [2] of the Technical Proposal

Manuscript received September 24, 2001.

The authors are with CERN-European Organization for Nuclear Research, CH-1211 Geneva 23, Switzerland.

Publisher Item Identifier S 1051-8223(02)03565-0.

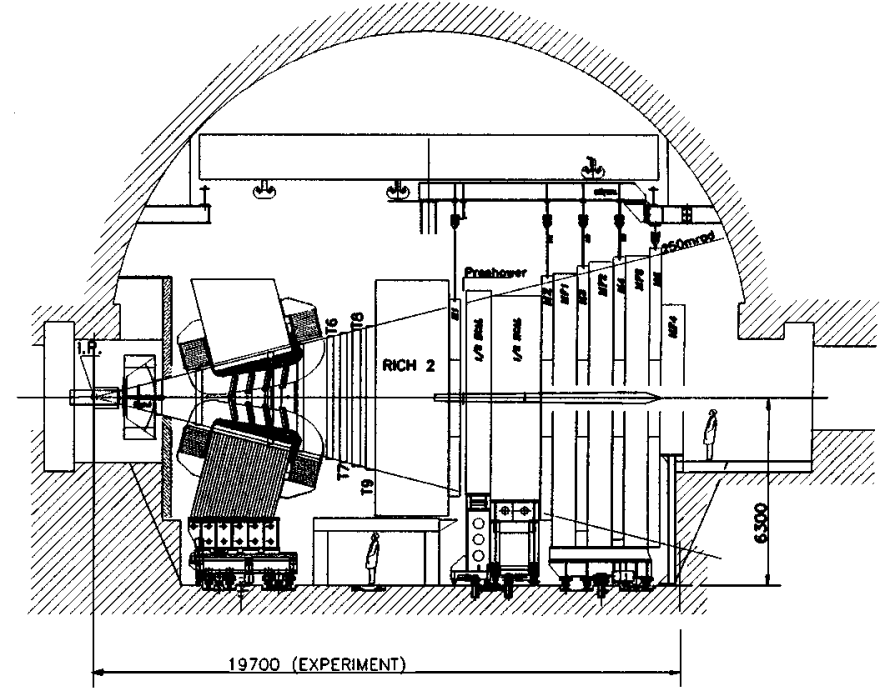

Fig. 1. Horizontal view of the $\mathrm{LHCb}$ detector in the underground experiment hall, seen from the center of the LHC ring (units in $\mathrm{mm}$ ).

with superconducting coils that required considerably higher investment costs and an unacceptably long construction time. Fig. 2 shows a perspective view of the magnet including its current and cooling water circuits. The main parameters are given in Table I.

The magnet consists of two trapezoidal coils bent by $45^{\circ}$ at the two transverse sides, arranged inside a window-frame yoke. The magnet gap is wedge-shaped in both the vertical and horizontal planes, following the detector acceptance. Large shims will be mounted along the pole faces outside the limits of the horizontal aperture. The $45^{\circ}$ bending of the coil sides together with the shims ensures a good homogeneity of the main field component across the gap, leading to a nonuniformity of the field integral to below $\pm 6 \%$.

To provide space for the frames of the tracking chambers positioned inside the magnet, the planes of the pole faces lie $100 \mathrm{~mm}$ outside the $\pm 250 \mathrm{mrad}$ vertical acceptance, and the shims on the side of the pole faces $100 \mathrm{~mm}$ outside the $\pm 300 \mathrm{mrad}$ horizontal acceptance. The coils, their clamps and support structure are mounted such that they do not penetrate into the clearance cone defined for the frames of the tracking chambers.

The large magnet gap implies relatively high stray fields in the detectors upstream and downstream of the magnet. The photosensitive detectors, hybrid photodiodes (HPDs), in the downstream Ring Image Cherenkov counter RICH2 allow local shielding, which is not possible for the upstream RICH1. Therefore shielding plates will be mounted close to the magnet at the upstream side to reduce the stray field at the RICH1 position to an acceptable level. 


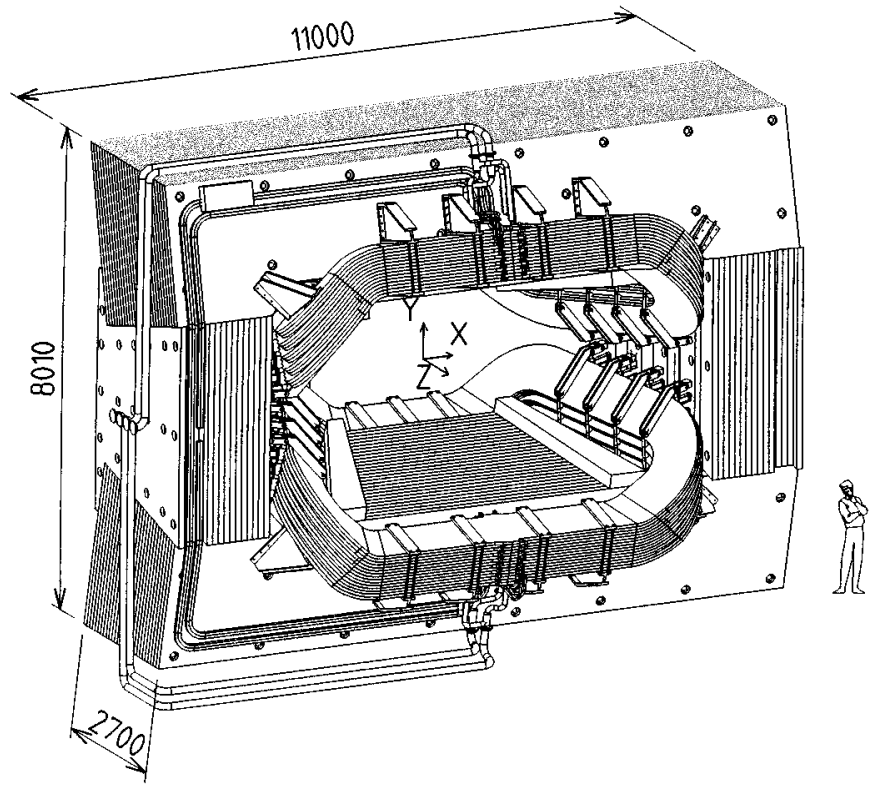

Fig. 2. Perspective view of the $\mathrm{LHCb}$ dipole magnet with its current and water connections. The coordinate system is also indicated (units in $\mathrm{mm}$ ).

\section{CONSTRUCTION OF THE EXCITATION COILs}

\section{A. General Coils Description}

The magnet is excited by two identical coils of conical saddle shape, mounted mirror symmetrically to each other in the magnet yoke. A coil is assembled from five subcoils (triplets), each one constructed from three monolayer pancakes. Each pancake is wound from a hollow extruded single-length conductor of about $310 \mathrm{~m}$ length. Pure aluminum Al-99.7 in an annealed state, having an ohmic resistance below $28 \mathrm{n} \Omega \mathrm{m}$ at $20{ }^{\circ} \mathrm{C}$ is chosen as conductor material. The conductor dimensions are listed in Table I.

The cross-section of the triplets is rectangular, also in the $45^{\circ}$ bent section. This is achieved by adjusting the transverse dimensions of the three pancakes accordingly during winding. Corresponding pancakes of successive sub-coils have identical dimensions when flat; they differ only in the winding direction to allow for easy serial current connection. Fig. 3 shows the assembly of a coil from triplets and the principal coil dimensions. All pancakes in a coil, and with this also all triplets, have the same bending radius at the $45^{\circ}$ bend. This implies a different setting of the bending line for each pancake in a coil but simplifies considerably the bending tool.

All electrical and hydraulic connections have to be at the downstream side of the magnet because of space restrictions between coils and shielding plates at the upstream side. All pancakes of the two coils are hydraulically in parallel, with the water inlet at the most downstream side. All water inlets and outlets will be connected to water collectors via manifolds and reinforced rubber tubes. The lateral thermal expansion of the coils during magnet operation is of the order of 4 to $5 \mathrm{~mm}$. The coil clamps will allow slight movement along the sub-coil planes to keep thermal stresses low. The electrical connections are located at the outer perimeter of the pancake ends. Solid gold-plated aluminum blocks of large contact area will be welded onto the
TABLE I

MAIN DESIGN PARAMETERS OF THE LHCb MAGNET

\begin{tabular}{|c|c|}
\hline Magnetic Parameters & \\
\hline Bending power & $\begin{array}{l}\int B \mathrm{dl}=4 \mathrm{Tm} \\
(10 \mathrm{~m} \text { track length })\end{array}$ \\
\hline Non-uniformity of $\int B \mathrm{dl}$ & $\begin{array}{l}\leq \pm 6 \% \text { in acceptance } \\
\text { (hor.: } \pm 300 \text { mrad, vert.: } \\
\pm 250 \mathrm{mrad})\end{array}$ \\
\hline Excitation current & $N I=2 \times 1.3 \mathrm{MA}$ \\
\hline Electric power dissipation & $P_{\mathrm{e}}=4.2 \mathrm{MW}$ \\
\hline Stored magnetic energy & $W_{\mathrm{m}} \approx 32 \mathrm{MJ}$ \\
\hline Inductance & $L \approx 2 \mathrm{H}$ \\
\hline Coils and Current & \\
\hline Coil construction (two coils) & 15 monolayer pancakes per coil \\
\hline Total number of turns & $N=2 \times 225$ \\
\hline Conductor material & Aluminium Al-99.7 \\
\hline Conductor dimensions & $50 \mathrm{~mm} \times 50 \mathrm{~mm}, \varnothing=25 \mathrm{~mm}$ \\
\hline Conductor length & $L_{\mathrm{c}} \approx 290 \mathrm{~m}$ per pancake \\
\hline Current in conductor & $I \approx 5.8 \mathrm{kA}$ \\
\hline Current density & $\sigma \approx 2.9 \mathrm{~A} / \mathrm{mm}^{2}$ \\
\hline Total resistance & $R=125 \mathrm{~m} \Omega @ 20^{\circ} \mathrm{C}$ \\
\hline Total voltage drop (two coils) & $U \approx 730 \mathrm{~V}$ \\
\hline Cooling & \\
\hline Cooling requirements & all pancakes in parallel \\
\hline Total water flow & $\phi \approx 150 \mathrm{~m}^{3} / \mathrm{h}$ \\
\hline Pressure drop of cooling water & $\Delta p \approx 10$ bar $@ \Delta T=24^{\circ} \mathrm{C}$ \\
\hline Mechanics & \\
\hline Steel quality of yoke & EN S235JRG2 \\
\hline Overall magnet dimensions & $H \times V \times L:$ \\
\hline $\begin{array}{l}\text { Useful wedged aperture } \\
\text { (upstream - downstream) }\end{array}$ & $\begin{array}{l}\text { hor: } 2.6-4.2 \mathrm{~m} \\
\text { vert: } 2.2-3.5 \mathrm{~m}\end{array}$ \\
\hline Weight of yoke & $M_{\mathrm{y}} \approx 1450 \mathrm{t}$ \\
\hline Weight of coils & $M_{\mathrm{c}} \approx 2 \times 25 \mathrm{t}$ \\
\hline
\end{tabular}

conductor ends in such a way that adjacent pancakes can be connected electrically in series.

\section{B. Pancake Winding}

The hollow conductor for the pancake winding is produced in a continuous way by rotary extrusion (Holton Conform ${ }^{\mathrm{TM}}$ ). In this process no billets have to be exchanged during the extrusion as is the case in a conventional extrusion press. Regions with extrusion welds along the conductor when going from one billet to the next, which might reduce some mechanical and electrical properties, are therefore excluded. The required continuous conductor length for winding one pancake is about $310 \mathrm{~m}$. It is delivered on a reel of $2 \mathrm{~m}$ inner diameter and tested for leaks and extrusion imperfections before being used for the pancake winding.

A pancake is wound around a mandrel, fixed on a turning table of an appropriate diameter. The mandrel determines the inner dimensions of the three different pancakes of a triplet. As the conductor is being unrolled off the reel, it is straightened before entering a brake unit. This is followed by a cleaning device with metallic brushes and vacuum suction, the taping unit to apply the glass fiber insulation, and a guidance unit to pass the conductor onto the turning table. All these devices are fixed on a common support with a pivot point upstream of the conductor reel. In this way the conductor stays tangential to the pancake circumference during winding. The brake holds the conductor 

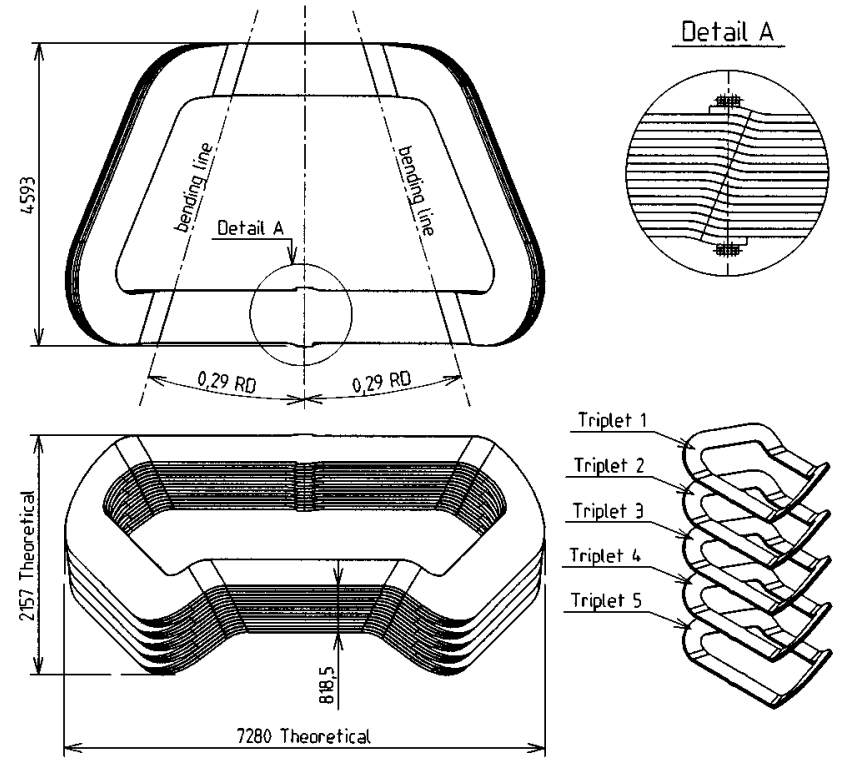

Fig. 3. Assembly of a coil from its triplets (units in $\mathrm{mm}$ ).

under constant mechanical tension to keep it aligned and straight during the winding process. The winding of the pancake is done with the glass fiber insulation tape around the conductor.

The turning table is able to turn clockwise and anticlockwise to allow the production of pancakes of opposite winding directions. Before winding a pancake, the starting end of the conductor is sealed, prepared for its water connection, and bent into a slight $\mathrm{S}$ shape, as shown in Fig. 3. Then the connector block for the electrical connection is welded on and fixed into a reference position on the mandrel. After a flat pancake is wound, the other conductor end is prepared accordingly with the connector block fixed and welded in a reference position.

It is only this central part of the mandrel, situated at the long straight section of a pancake, which has to be inverted when going from clockwise to anticlockwise winding. Each individual flat pancake is wrapped after winding with glass fiber tape and fixed in its shape by special clamps. Afterwards, the corresponding pancakes that form one triplet are bent by $45^{\circ}$, placed into the mold and vacuum impregnated.

The smallest winding and bending radius is $400 \mathrm{~mm}$ for all pancakes. The radius is large in order to keep the keystone deformation below $1 \mathrm{~mm}$. The height of the mold and the thickness of the glass fiber insulation are chosen in relation to the keystone deformation and to guarantee a good electrical insulation. The minimum ground insulation thickness of a triplet is $3 \mathrm{~mm}$.

\section{Coil Assembly and Main Production Tests}

The assembly of a coil from sub-coils is shown in Fig. 3. Special care is required for the handling of the individual triplets. Successive triplets are separated by a layer of $1 \mathrm{~mm}$ thick soft insulator to compensate for inaccuracy in the triplet manufacturing. Clamps are used to press the sub-coils together and to handle each coil as one unit for transportation. The same clamps serve to fix the coils onto the magnet yoke.

After the coil assembly, successive electrical connection blocks have to be screwed tightly together to guarantee a very good electrical contact between adjacent pancakes. To provide some mechanical flexibility for this connection, the last $50 \mathrm{~cm}$ of the two conductor ends of the pancakes are not molded to the neighboring turns. Gold-plated distance sheets will be inserted to connect the blocks between successive triplets.

Before extruding continuous conductor pieces of $310 \mathrm{~m}$ length for the pancake winding, long-enough test pieces were extruded to analyze the mechanical and electrical properties and to determine the relevant parameters for setting up the extrusion machine. All these parameters are monitored and kept constant during the production extrusion. Each reel with $310 \mathrm{~m}$ of conductor is leak tested with 50 bar water pressure at the extrusion factory and before its use for the pancake winding. This pressure test is repeated for each triplet and at each assembled coil.

Turn-to-turn insulation tests are performed during the pancake winding. The insulation between pancakes and to ground is verified before and after the vacuum impregnation of each triplet. The resistance of each pancake is measured. After the coil assembly, the total resistance is checked for consistency.

\section{MAGNET YOKE}

The magnetic flux generated by the two coils is shaped and guided by the steel yoke in the useful volume required by the detector acceptance. The yoke has been designed as windowframe type with small cut-outs for the coils. It is assembled from identical horizontal bottom and top parts and two identical vertical parts (uprights) to close the flux return, see Fig. 2. It produces an almost vertical field in the wedge-shaped gap between the pole faces. Shims at the sides of the pole faces will improve the homogeneity of the main field component. The exact shape and dimensions of the shims have still to be optimized.

The design of the yoke has to respect the boundary conditions given by the existing cavern at pit 8 of the LHC accelerator ring and its infrastructure. The hall is equipped with two cranes, each of $40 \mathrm{t}$ lifting capacity and of restricted lateral displacement. For reasons of cost and transportability by road, the magnet yoke parts will be assembled from industrial standard, rolled, low carbon steel plates of $100 \mathrm{~mm}$ thickness of designation EN-S235JRG2. The maximum weight of a single plate will not exceed $25 \mathrm{t}$. It is intended to re-use the existing rail system, embedded in the concrete floor of the hall, for the magnet assembly and displacement.

The steel plates of the bottom and top part of the yoke are arranged orthogonal to the planes which limit the vertical aperture to $\pm 250 \mathrm{mrad}$. The stronger magnetic field at the upstream side of the aperture requires there a larger amount of steel in the horizontal and vertical yoke parts than at the downstream side. This is achieved by having the pole faces and the inner side-walls of the uprights follow the wedge-shaped aperture, whereas the outer walls of the yoke are rectangular and parallel to the beam.

\section{MAGNet ASSEMbly IN THE UndergRound EXPERIMENT HALL}

The position of the magnet in the experiment is not reachable by the crane. The magnet has therefore to be assembled on a 

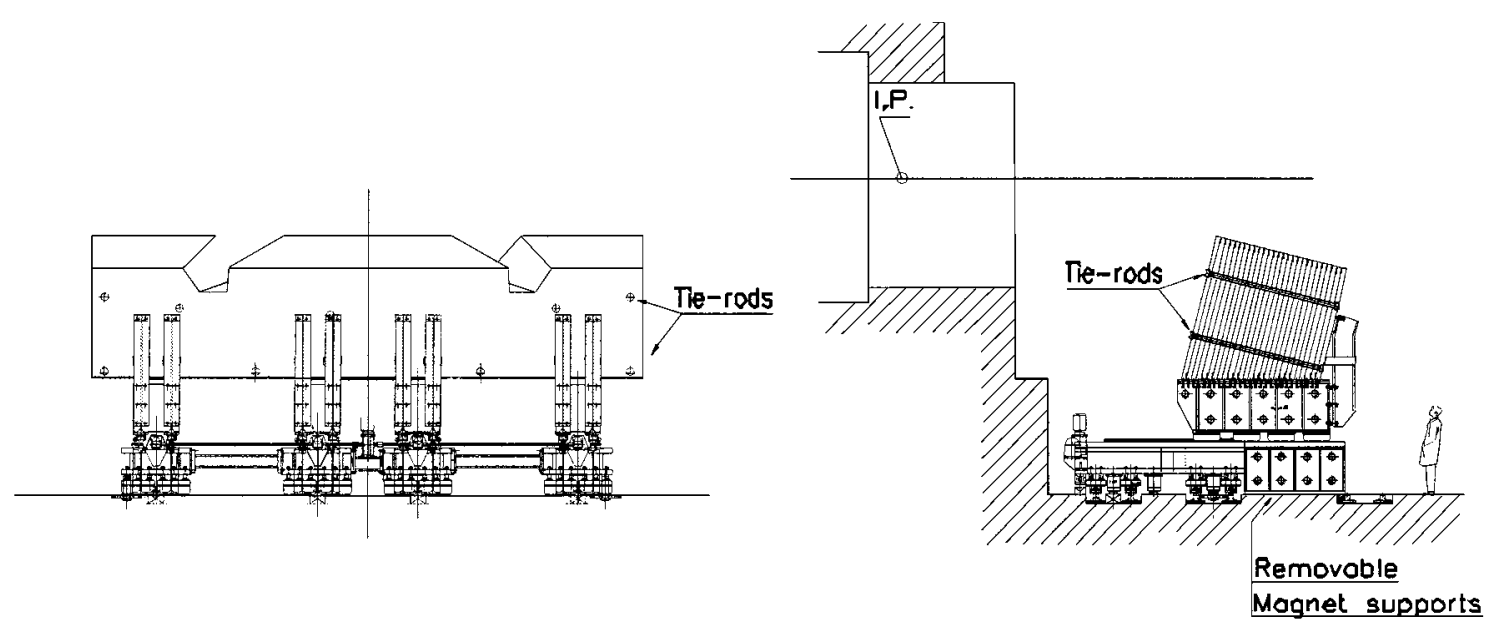

Fig. 4. Assembly of the lower part of the magnet yoke in the underground hall, looking toward the interaction point (left) and seen from the side (right).

carriage in a place covered by the crane and will then be rolled laterally and in the beam direction into its final place. It is foreseen to re-use the DELPHI magnet carriage on its rail system as a platform for the $\mathrm{LHCb}$ magnet.

The horizontal beams and the uprights of the magnet yoke will be assembled from individual $100 \mathrm{~mm}$ thick steel plates. Large tie-rods will be used to consolidate the different yoke parts. The plates are flame-cut along the outer yoke faces. Only the mating faces between the bottom and top yoke parts and the uprights and the pole faces are machined. Special jigs will be temporarily mounted on the carriage to support the steel plates of the bottom horizontal yoke part during assembly. The assembly starts with the smallest plate. The next larger plate will be placed against it. Each plate can be adjusted individually in height such that the mating surfaces with the uprights are flat. Adjacent plates will be spot-welded together temporarily to avoid their sliding off. After having assembled all 27 plates, large M80 tie-rods will pull the plates together. Each tie-rod has a pulling force of $200 \mathrm{t}$. The spot-welds will be removed during the tensioning of the tie-rods. The bottom yoke part will now be fixed solidly to the carriage and underfilled with nonshrinkable cement. Fig. 4 shows the bottom yoke part with its temporary jigs in its mounting position on the carriage.

The assembly of the uprights will follow next. Temporary jigs will be used to fix the outermost plate in the vertical position. During the assembly, the plates have to be secured against falling over. When the correct number of sheets for each thickness step of the uprights has been reached, the corresponding tie-rods will be mounted and pre-stressed. After all the plates of an upright have been mounted, the remaining tie-rods will be mounted and all tie-rods stressed to consolidate the block.

All tolerances in machining the individual plates of the uprights appear as nonflatness of the upper mating surfaces. The plates will be shimmed with soft steel strips of appropriate thickness.

Next the coils have to be mounted, each one fully equipped and tested with its electrical and hydraulic connections and controls sensors. The lower coil will be put in place and fixed first. Its position serves as reference to prepare the mounting of the

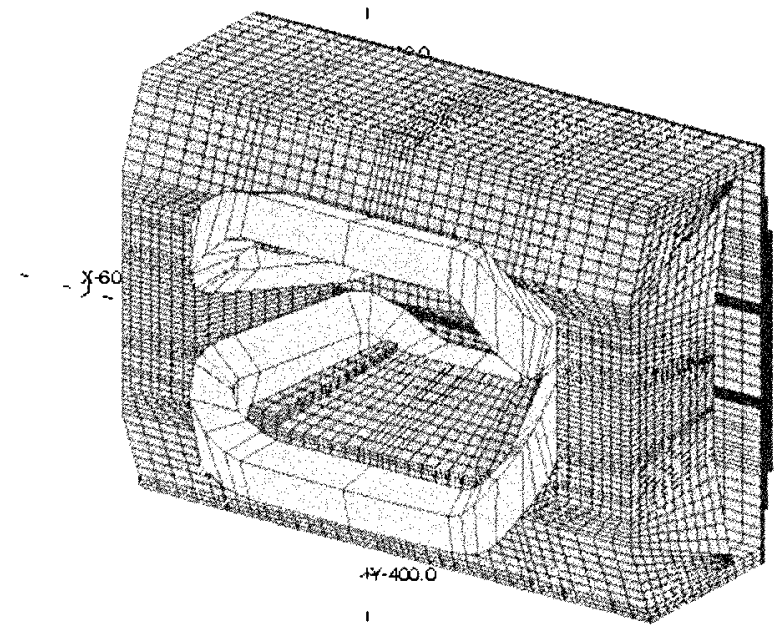

Fig. 5. The TOSCA model of the LHCb magnet with its shielding plates.

upper coil. Next the upper coil will be placed into the magnet and provisionally supported in a position as low as possible. This ensures the safe introduction of the lower row of tie-rods in the top yoke part. The top yoke part will be assembled using temporary jigs and the plates solidly pulled together by the large tie-rods. Afterwards the upper coil has to be fixed in its final position and the shims mounted. The relative position of the four yoke parts to each other will be assured by welding solid clamping brackets onto the different parts, each with appropriate dowel pins.

Next the shielding plates will be mounted. They are supported by the magnet carriage and fixed onto the yoke. After completion the magnet will be rolled into its final position, connected to the water and current distribution, and commissioned.

Straight after commissioning the field will be mapped. Special attention has to be given to the fact that the steel in the nearby concrete will guide flux lines to unwanted places, e.g., to the region near RICH1. The field has therefore to be measured also in such regions to allow the installation of local additional shielding plates on the floor and the surrounding walls before the installation of the detectors. 


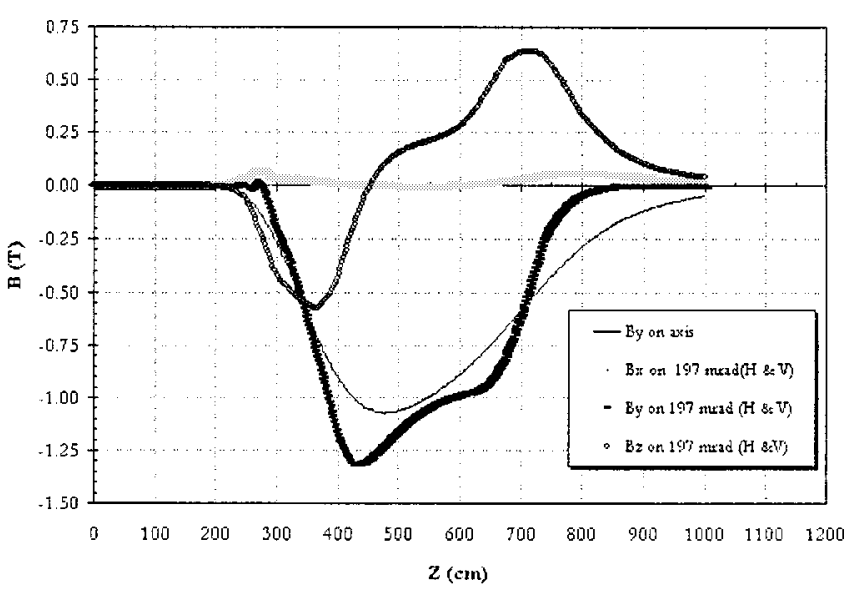

Fig. 6. Components of magnetic flux density along selected tracks.

\section{Field Calculations and Shielding Plates}

Finite-element calculations were used in the magnet design. First results were obtained in 2-D computations using the POISSON program. These calculations were followed by full 3-D analysis, mainly with the TOSCA program, but also with ANSYS for the electromagnetic and thermal stress calculations. Measured $B-H$ curves for standard ENS235JRG2 steel were used for all TOSCA computations.

A full TOSCA 3-D model of the magnet is shown in Fig. 5. Models with up to 275000 nodes were used to study saturation effects in the magnet yoke, field inhomogeneities, field integrals in the tracking volume, and stray field estimates. The large gap size of the magnet creates unwanted stray fields outside the useful volume, which influences the functioning of some detectors. The largest effects are on the hybrid photodiodes (HPDs) of the first Ring Imaging Cherenkov counter (RICH1), situated close to the magnet on the upstream side. Without any shielding this field is of the order of $0.026 \mathrm{~T}$ at the HPDs. With shielding plates of total thickness of $300 \mathrm{~mm}$, as indicated in Fig. 5, it can be reduced to below $0.003 \mathrm{~T}$. The shielding plates have to be composed of two halves with a vertical cut along the beam line, where one half can be rolled out to give free access to the beam vacuum chamber.

The uniformity of the field integral of the principal (vertical) component $B_{y}$ is of greatest relevance to the experiment. It is evaluated along straight tracks originating at the interaction point. The variations relative to the central track are below $\pm 6 \%$. The homogeneity of the main field components across the gap and of the field integral depends strongly on the geometry of the shims. They were roughly optimized using the POISSON program. Further optimization is possible.

The variation of the main field component along the beam direction $z$ is shown in Fig. 6 for a central track and of the three field components for a track at $197 \mathrm{mrad}$ (in $x$ and $y$ ). A current of 1.2 MA per coil has been assumed for this calculation.

The components of the Lorentz forces and of the forces due to thermal stresses during the operation of the coils are important for the design of the coil supports in the magnet. The largest Lorentz force is at the upstream part of the coils. The total force there is about $90 \mathrm{t} / \mathrm{m}$, with the largest component of $70 \mathrm{t} / \mathrm{m}$ parallel to the steel plates in the up (down) direction for the upper (lower) coil. All pancakes of the two coils are hydraulically connected in parallel. To contain the high thermal stresses in the impregnated pancakes, the water inlet for all pancakes is at their outer perimeter at the downstream side of the coils. In this way the outer cold turns of a pancake act as a belt around the inner warm turns. The thermal forces act in the plane of the pancake and try to increase the plane dimensions. For free thermal expansion of the pancakes under these conditions, the maximum thermal stress is at the smallest winding radius of the pancake. For a temperature difference between inlet and outlet cooling water of $\Delta T=30{ }^{\circ} \mathrm{C}$ the maximum stress is $\sigma \approx 25 \mathrm{MPa}$. The coil supports have been designed to discharge the Lorentz forces to the magnet yoke, and the coil clamps have to allow for thermal expansion in the plane of the pancakes.

\section{SUmmary OF Status AND PlanNing}

The construction of the magnet is financed by the Common Fund of the LHCb Collaboration. The reference design, laid down in the Magnet Technical Design Report [1], was approved by the LHC Committee in April 2000. Since then the magnet components have been tendered and construction has started. Three separate contracts were placed: a) for the extrusion of the aluminum conductor; b) for the construction of the coils; and c) for the manufacture of the steel plates for the yoke. The Tendering Documents for the power supply were sent out in June 2001. Smaller contracts for the magnet support, jigs, and handling devices will follow soon.

Prototype extruded conductor pieces, cut to $6 \mathrm{~m}$ length, have been delivered to CERN and tested for mechanical dimensions and properties, for specific electric resistance and chemical composition. A conductor of $120 \mathrm{~m}$ length of final properties was delivered in September 2001 to the coil production firm. The final conductor pieces, each of $310 \mathrm{~m}$ length on one large-diameter reel, will be extruded and transported to the coil producer in 3 batches of 10 reels.

The coil producer has increased its production facilities to allow the manufacture, handling, and assembly of these large coils. It is foreseen to wind one dummy pancake with the $120 \mathrm{~m}$ pre-extrusion in October 2001. This test production should validate the complete production chain, including the pancake winding, the $45^{\circ}$ bending, the triplet molding; as well as the handling tools. The delivery of the two coils to CERN is scheduled for December 2002.

The production of the yoke plates is straightforward. The plate manufacturer has reserved the required quantity of quality steel in the rolling mill. Test pieces of these plates will be delivered to CERN in autumn 2001 for verification of the magnetic and mechanical properties. After successfully passing the quality criteria, the flame-cutting and machining of the plates could start in December 2001. The total delivery will then be finished in summer 2002.

The magnet will be assembled in the first half of 2003 and the field measured in autumn 2003. 


\section{ACKNOWLEDGMENT}

The authors would like to thank the LHCb Collaboration for their continuous support and the many discussions with and strong encouragement of its technical co-ordinator H. J. Hilke.

\section{REFERENCES}

[1] "LHCb magnet technical design report 1,", CERN document CERN/LHCC 2000-007, Dec. 17, 1999.

[2] "LHCb, A large hadron collider beauty experiment, Technical Proposal,", CERN document CERN/LHCC 98-4, Feb. 20, 1998. 